\title{
Long noncoding RNA-HOTAIR affects chemoresistance by regulating HOXA1 methylation in small cell lung cancer cells
}

\author{
Shun Fang ${ }^{1,3}$, Hongyi Gao ${ }^{1,2,3}$, Yue Tong ${ }^{1}$, Jie Yang ${ }^{1}$, Ruixiang Tang ${ }^{1}$, Yuchun Niu', Man Li ${ }^{1}$ and Linlang Guo ${ }^{1}$
}

Homeobox (HOX) transcript antisense RNA (HOTAIR), a long intergenic noncoding RNA (lincRNA), has been reported to play an oncogenic role in various cancers including small cell lung cancer (SCLC). However, it is not known whether HOTAIR can modulate chemoresistance in SCLC. The aim of this study is to investigate the roles of HOTAIR in chemoresistance of SCLC and its possible molecular mechanism. Knockdown of HOTAIR was carried out in SCLC multidrug-resistant cell lines (H69AR and H446AR) and the parental cell lines (H69 and H446) to assess its influence on chemoresistance. The results showed that downregulation of HOTAIR increased cell sensitivity to anticancer drugs through increasing cell apoptosis and cell cycle arrest, and suppressed tumor growth in vivo. Moreover, HOXA1 methylation increased in the resistant cells using bisulfite sequencing PCR. Depletion of HOTAIR reduced HOXA1 methylation by decreasing DNMT1 and DNMT3b expression. The interaction between HOTAIR and HOXA1 was validated by RNA immunoprecipitation. Taken together, our study suggested that HOTAIR mediates chemoresistance of SCLC by regulating HOXA1 methylation and could be utilized as a potential target for new adjuvant therapies against chemoresistance.

Laboratory Investigation (2016) 96, 60-68; doi:10.1038/labinvest.2015.123; published online 2 November 2015

Lung cancer is one of the leading malignant tumors in the world. Small cell lung cancer (SCLC) accounts for $\sim 15 \%$ of all lung cancers at present. First-line chemotherapy based on cisplatin is the current optimal treatment for SCLC. ${ }^{1}$ SCLC is characterized by a propensity for early dissemination and rapid development of drug resistance during the course of disease, although patients have initial high response rates to chemotherapy. Several novel molecules are presently undergoing evaluation, and represent potential future additions to the treatment repertoire for SCLC. ${ }^{2}$ However, the chemoresistance of SCLC remains an important issue worldwide. ${ }^{1}$

Long noncoding RNAs (lncRNAs) have been demonstrated to regulate nearly every step in the life cycle of a gene over the past few years. ${ }^{3}$ Several lncRNAs may also be involved in oncogenesis and development of some kinds of cancers such as leukemia, lung cancer, and hepatocarcinoma. ${ }^{4}$ Recent studies showed that some lncRNAs such as BX648420, ENST00000366408, and AK126698 were correlated to resistance to cisplatin in NSCLC. AK126698 appeared to confer cisplatin resistance by targeting the Wnt pathway. ${ }^{5}$
LncRNA-HOTAIR, identified from a custom tilling array of the HOXC locus (12q13.13; Puglisi et $\left.a l^{2}\right){ }^{6}$ can promote tumor progression such as colorectal cancer, pancreatic cancer, and NSCLC. Its molecular mechanism was reported to silence targeted genes such as HOXD10 and PGR by inducing genome-wide retargeting of polycomb-repressive complex 2 (PRC2), which was composed of EZH2, SUZ12, and EED, leading to the methylation of H3K27.7 Homeobox (HOX) transcript antisense RNA (HOTAIR) has also been reported to affect cellular proliferation, invasiveness, and clinical relapse in SCLC. ${ }^{8}$ However, the involvement of lncRNA-HOTAIR in chemoresistance of SCLC remains unknown.

The aberrant DNA methylation has been well described as a novel marker for cancer diagnosis, prognosis, and prediction of response to chemotherapeutic drugs. In glioma, the hypermethylation at MGMT indicated resistance to a chemotherapeutic agent, whereas in breast cancer, the methylation of BRCA1 was indicative of successful chemotherapy. ${ }^{9}$ In NSCLC, SULF2 and IGFBP-3 have also been observed to affect drug sensitivity of NSCLC cells. ${ }^{10-12}$

\footnotetext{
${ }^{1}$ Department of Pathology, Zhujiang Hospital, Southern Medical University, Guangzhou, China and ²Department of Pathology, Guangdong Provincial Maternity and Child Care Center, Guangzhou, China

Correspondence: Professor L Guo, MD, PhD, Department of Pathology, Zhujiang Hospital, Southern Medical University, 253 Gongye Road, Guangzhou 510282, China. E-mail: linlangg@yahoo.com

${ }^{3}$ These two authors contributed equally to this work.

Received 26 January 2015; revised 14 August 2015; accepted 2 September 2015
} 
The HOX family, including 39 HOX genes, is divided into four groups $(\mathrm{A}-\mathrm{D})$ in tightly linked clusters on different chromosomes, and has the equivalent position in each cluster. HOXA1, one of the HOX family members, has been reported to be involved in many important biological processes including cell growth, apoptosis, transformation, and survival. ${ }^{13}$ Our previous study showed that HOXA1, targeted by miR-100, played a role in SCLC cell survival and chemoresistance. ${ }^{14}$ HOXA1 was found to stimulate STAT3 and STAT5B in oncogenic transformation of the immortalized mammary epithelial cells, ${ }^{15}$ and regulated the p44/42 MAP kinase pathway in mammary cancer cells. ${ }^{16}$ The overexpression of HOXA1 was associated with poor outcome in patients with HCC, whereas the downregulation of HOXA1 can inhibit cell growth, anchorage-independent growth, migration, and invasion of HepG2 cells. ${ }^{17}$

In the present study, we first detected the level of lncRNAHOTAIR in SCLC multidrug resistance cells (H69AR and H446AR) and found the overexpression of HOTAIR in H69AR and H446AR cells. Next, we investigated the biological functions of lncRNA-HOTAIR in SCLC, in vitro and in vivo. Knockdown of HOTAIR increased cell sensitivity to anticancer drugs, cell apoptosis, and inhibited tumor growth. Moreover, we found that HOTAIR modulated HOXA1 methylation, through decreasing DNMT1 and DNMT3b expression. These findings revealed a novel mechanism by which HOTAIR mediated chemoresistance of SCLC.

\section{MATERIALS AND METHODS}

\section{Cell Culture}

The human small cell lung cancer cell lines NCI-H446 and NCI-H69 and the drug-resistant subline H69AR were purchased from American Type Culture Collection (ATCC, USA), and the drug-resistant subline H446AR was constructed by exposing at intervals and repeatedly to high dose of adriamycin (ADM). ${ }^{18}$ All cell lines were maintained in RPMI-1640 medium with $10 \%$ fetal calf serum in an incubator at $37^{\circ} \mathrm{C}$ with $5 \% \mathrm{CO}_{2}$. $\mathrm{H} 69 \mathrm{AR}$ and $\mathrm{H} 446 \mathrm{AR}$ were alternately fed with drug-free medium and medium containing $0.8 \mu \mathrm{M}$ of ADM. The resistant cell lines were tested regularly for maintaining resistance to the selected drugs. Growth and morphology of all cell lines were monitored on a weekly basis.

\section{Small Interference RNA (siRNA), Short Hairpin RNA (shRNA), and Transfection}

The IncRNA-HOTAIR siRNA and HOXAl siRNA were purchased from Sigma Aldrich (Shanghai, China), and the oligonucleotides encoding siRNA were as follows: siHOTAIR-1115: 5'-CCCAUGGACUCAUAAACAATT- $3^{\prime}$ and $5^{\prime}$-UUG UUUAUGAGUCCAUGGGTT-3'; siHOTAIR-1560: 5'-GC- C UUUGGAAGCUCUUGAATT- $3^{\prime}$ and $5^{\prime}$-UUCAAGAGCUUC CAAAGGCTT-3'. Lipofectamine 2000 was purchased from Invitrogen (Shanghai, China) and siRNA transfection was done according to the protocol supplied by Invitrogen.
To stably silence endogenous HOTAIR, two shRNA oligonucleotides were purchased from Sigma Aldrich (Shanghai, China), and the oligonucleotides were as follows: shHOTAIR-I: 5'-CACCGCCTTTGCTTCGTGCTGATTCCGAAGAATCAG CACGAAGCAAAGGC- $3^{\prime}$ and $5^{\prime}$-AAAAGCCTTTGCTTCGT GCTGATTCTTCGGAATCAGCACACGAAGCAAAGGC-3';11 shHOTAIR-II: 5'-GATCCCGAACGGGAGTACAGAGAGATT CAAGAGATCTCTCTGTACTCCCGTTCTTTTTTGGAAA-3' and 5'-CGCGTTTCCAAAAAAGAACGGGAGTACAGAGAG ATCTCTTGAATCTCTCTGTACTCCCGTTCGG- $3^{\prime} ;^{12}$ they were cloned into the pGCSi-neo-GFP vector to generate pGCSi-neo-GFP-HOTAIR-RNAi(s). SCLC cells were transfected with shRNA via Lipofectamine 2000 (Invitrogen). Cells were sorted at $24 \mathrm{~h}$ after transfection by GFP expression on a FACSAria cell sorter machine that produced a population that was $80 \%$ GFP positive. At $48 \mathrm{~h}$, cells were exposed to G418 (400 ng/ml, Sigma-Aldrich, St Louis, MO, USA) selection for 1 month. G418-resistant cells were ring-cloned and expanded to establish stable shHOTAIR and shNC transfectants.

\section{Drug-Resistance Assay}

In vitro drug cytotoxicity was measured by Cell Counting Kit-8 (CCK-8) assays. The cells incubated without drugs (i.e., control wells) were set at 100\% survival and were used to calculate the concentration of each cytostatic drug lethal to $50 \%$ of the cells $\left(\mathrm{IC}_{50}\right)$. The ranges of drug concentrations were based on earlier studies and aimed at obtaining an $\mathrm{IC}_{50}$ value for all cases. A total of three anticancer drugs (Cisplatin (CDDP; Shandong, China), Adriamycin (ADM; Jiangsu, China), and Etoposide (VP-16; Jiangsu, China)) were obtained from commercial sources and were dissolved according to the manufacturer's instructions and tested in eight concentrations. Cells were seeded into 96-well plates (4000 cells/well) and then treated for $24 \mathrm{~h}$ in $100 \mu \mathrm{l}$ of medium with anticancer drugs. CCK-8 reagent (Dojindo, Kumamoto, Japan) was then added and the cells were incubated at $37^{\circ} \mathrm{C}$ for $4 \mathrm{~h}$ before reading the absorbency using a microplate reader ( $\mu$ Quant, Bio-Tek Instruments) at $450 \mathrm{~nm}$. The assay was conducted in five replicate wells for each sample and three parallel experiments were performed.

\section{Flow Cytometry}

For cell cycle analysis, cells were seeded in six-well plates $\left(1 \times 10^{6}\right.$ cells /well $)$ and then treated for $24 \mathrm{~h}$ in $2 \mathrm{ml}$ of medium with anticancer drugs or mock as described above. The cells were washed twice in cold PBS buffer and fixed with $70 \%$ ethanol. After centrifugation at $2000 \mathrm{~g}$ for $5 \mathrm{~min}$, the cells were then treated with $0.1 \mathrm{mg} / \mathrm{ml}$ RNase A (Boehringer Mannheim) and stained with $50 \mathrm{mg} / \mathrm{ml}$ propidium iodide (Sigma Chemical). All samples were prepared following the manufacturer's instructions and analyzed by flow cytometry (FACSCalibur Becton Dickinson) using CellQuest software (BD Bioscience). For apoptosis assays, cells were seeded in six-well plates $\left(1 \times 10^{6}\right.$ cells/well $)$ and then treated for $24 \mathrm{~h}$ in $2 \mathrm{ml}$ of medium with anticancer drugs or mock as described 
above, and were washed twice in cold PBS buffer and fixed with $70 \%$ ethanol. Apoptosis was detected by Annexin V-fluorescein isothiocyanate (FITC).

\section{In Vivo Tumor Xenograft Model}

Male BALB/c nude mice aged 4-5 weeks were purchased from the Laboratory Animal Centre of Zhongshan University (Guangzhou). All procedures were performed according to the internationally accepted ethical guidelines. The BALB/c nude mice were randomly assigned to groups ( $n=5 /$ group). For the tumorigenicity studies, cells were harvested, washed with PBS, and resuspended in normal culture medium at a concentration of $1 \times 10^{7}$ cells per $0.2 \mathrm{ml}$. Each subculturing situs was injected with viable $1 \times 10^{7}$ cells per $0.2 \mathrm{ml}$ in the back leg to establish the tumor model, respectively. At 1 month after injection, all the mice were killed. The tumors were excised. Tumor volumes were calculated using the ellipsoid volume formula $\left(0.5 \times l \times w^{2}\right.$, where $l$ is length and $w$ is width).

\section{Bisulfite Sequencing PCR (BSP)}

DNA from SCLC cell lines were isolated, bisulfate modified, and then used for BS analysis as previously described. ${ }^{13}$ Primer sequences for PCR were as follows: BSP-HOXA1-F: 5'-GT TTAGTTTTAGAATAGAGGAGGTGGTT-3'; BSP-HOXA1-R: 5'-AAAATCAATAAACCAA AACTC-3'. The region of HOXA1 promoter is shown in Supplementary Figures S1 and S7.

\section{Bioinformatics-Based Tool}

We used catRAPID algorithm to compute the interaction propensity of HOXA1 protein with lncRNA-HOTAIR. CatRAPID predicts protein-RNA associations by estimating the interaction propensity between amino acids and nucleotides using secondary structure information, hydrogen bonding, and Van der Waals forces. The approach was previously applied to predict associations between different types of proteins and RNA molecules. Although each protein binds to distinct types of RNA structures, we observe that the contribution of hairpin loops accounts for $57 \%$ of the overall interaction propensity.

\section{Quantitative RT-PCR}

Our observations were made in an ABI Prizm 7300 Sequence Detection System using SYBR Green (Applied Biosystems, Foster City, CA, USA) following the manufacturer's instructions. Primers for quantitative RT-PCR were designed based on sequences from the GenBank as follows:

HOTAIR: F: 5'-GGTAGAAAAAGCAACCACGAAGC-3', R: 5'-ACATAAACCTCTGTCTGTGAGTGCC-3'; HOXA1: F:
5'-AGTTGGAGAGTACGGCTACCTG-3'， R: 5'-TGCAGGGA TGCAGCGATCTCCAC-3'; GAPDH: F: 5'-GAGTCAACGGAT TTGGTCGT-3'， R: 5'-CATGGGTGGAATCATATTGGA-3' . The relative mRNA expression level was calculated by using the comparative expression level $2^{-\Delta \Delta \mathrm{CT}}$ method.

\section{Western Blotting Analysis}

Protein extract $(30 \mu \mathrm{g})$ was separated in a $15 \%$ SDS-polyacrylamide gel and electrophoretically transferred onto a PDVF membrane (Millipore, Etten-Leur, The Netherlands). Membranes were blocked overnight with 5\% non-fat dried milk and incubated for $2 \mathrm{~h}$ with an anti-HOXA1 antibody (Sigma, USA) or anti-GAPDH antibody (Sigma, USA). After washing with TBST $(10 \mathrm{mM}$ Tris, $\mathrm{pH} 8.0,150 \mathrm{mM} \mathrm{NaCl}$, and $0.1 \%$ Tween-20), the membranes were incubated for $1 \mathrm{~h}$ with horseradish peroxidase-linked goat-anti-rabbit antibody (KPL, Gaithersburg, MD, USA). The membranes were washed again with TBST, and the proteins were visualized using ECL chemiluminescence.

\section{RNA Immunoprecipitation}

We performed RNA immunoprecipitation (RIP) method using Magna RIP RNA-Binding Protein Immunoprecipitation Kit (Millipore) according to the manufacturer's instructions. The HOXA1 antibody (Santa Cruz, USA) was used for RIP. The coprecipitated RNA was detected by reverse transcription-PCR and quantitative PCR. The primer sequences were listed as above. Total RNA (input controls) and isotype controls were assayed simultaneously to demonstrate that the detected signals were the result of RNA specifically binding to HOXA1.

\section{Enzyme-Linked Immunosorbent Assay (ELISA)}

For DNA methyltransferase (DNMT) measurement, $100 \mu \mathrm{l}$ standard or samples were added to each well of 96-well plates. After incubation for $2 \mathrm{~h}$ at $37^{\circ} \mathrm{C}$, reagents were added to the wells according to the manufacturer's instructions of ELISA kit (GENMED DNMT total activity kit, Genmed Scientifics, USA). A microplate reader ( $\mu$ Quant, Bio-Tek Instruments) was used to read the absorbency at $450 \mathrm{~nm}$. The duplicate readings were averaged. Software curve expert 1.30 was used to construct a standard curve and calculate the concentration of each sample.

\section{Statistical Analysis}

The data are presented as means \pm s.d. Data of related assay were assessed by one-way ANOVA. SPSS 16.0 software (SPSS, Chicago, IL, USA) was used for all statistical analyses, with $P<0.05$ considered statistically significant.

Figure 1 LncRNA-HOTAIR is required for refractoriness to treatment with anticancer drugs. (a) RT-PCR analysis of IncRNA-HOTAIR expression in resistant cells (H69AR and H446AR) and their parental cells (H69 and H446). (b) Downregulation of IncRNA-HOTAIR by siRNA was validated by RT-PCR. (c) IC 50 was measured by CCK-8 assay when IncHOTAIR-downregulated cells were exposed to CDDP, ADM, and VP-16. (d and e) Cell early apoptosis and cell cycle progression were determined by flow cytometric analysis in IncHOTAIR-downregulated cells after drug exposure. Results are mean \pm s.d. ${ }^{*} P<0.05$, ${ }^{* *} P<0.01$. 
a
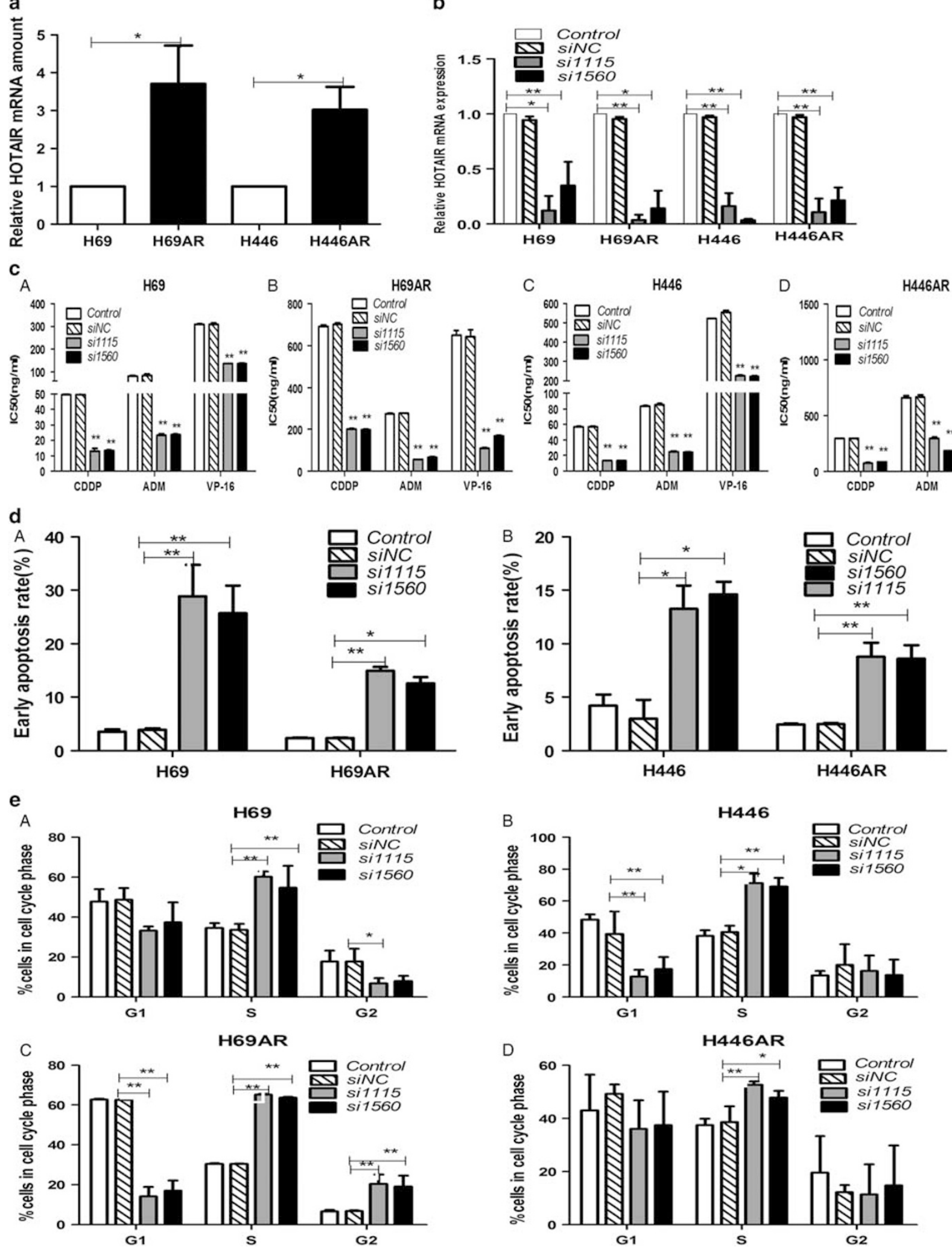


\section{RESULTS}

HOTAIR Knockdown Increased Drug Sensitivity In Vitro

We tested IncRNA-HOTAIR expression by RT-PCR in H69/ H69AR and H446/H446AR cells and found that the expression was elevated by 2.8-3.6-fold in the resistant cells compared with the parental cells (Figure 1a). To confirm the role of lncRNAHOTAIR in multidrug resistance of SCLC, we performed CCK-8 assay and flow cytometry to test the effect of the lncRNA-HOTAIR on cell sensitivity to anticancer drugs, cell apoptosis, and cell cycle progression in H69, H69AR, H446, and H446AR cells. We repressed the lncRNA-HOTAIR expression by siRNA and showed the expression was downregulated by 35-97-fold (Figure 1b). CCK-8 assays showed that $\mathrm{IC}_{50}$ values of CDDP, ADM, and VP-16 decreased significantly after transfection with siHOTAIR (Figure 1c).

CDDP and VP-16 should induce DNA damage, and result in S-phase arrest. To investigate the possible mechanisms of HOTAIR in chemoresistance, we evaluated the impact of HOTAIR on apoptosis and cell cycle control when cells were exposed to chemotherapeutic drugs. Flow cytometry results showed that the apoptosis rates increased significantly in the lncRNA-HOTAIR-downregulated cells after drug exposure (Figure 1d), the $S$ phase was prolonged, and the cell cycle progression was delayed after drug exposure (Figure 1e; cell apoptosis rates and cell cycle progression upon CDDP and VP-16 exposure are given in Supplementary Figures S2 and S3). These observations suggested that knockdown of
lncRNA-HOTAIR increased drug sensitivity, enhanced cell apoptosis, and decelerated cell cycle progression.

\section{HOTAIR Knockdown Suppressed Tumor Growth In Vivo}

To examine the effect of lncRNA-HOTAIR on tumor growth, we subcutaneously injected SCLC cells transfected with shRNAs specific against lncRNA-HOTAIR or negative control (NC) into nude mice. Mice were killed after 4 weeks. The tumors of shHOTAIR-treated cells grew significantly slowly compared with those of shNC-treated cells (Figure $2 \mathrm{a}$ and $\mathrm{c}$ ). Growth curves showed the tumor volumes of shHOTAIRtreated cells decreased significantly (Figure $2 \mathrm{~b}$ and $\mathrm{d}$ ).

\section{Methylation Status of HOXA1 Was Associated with Chemoresistance of SCLC}

DNA hypermethylation has been found to be one of the mechanisms in activating genes required for drug cytotoxicity in the resistant phenotype development. ${ }^{9}$ In this study, we found that the methylation levels of HOXA1 were higher in resistant cells than in parental cells. Meanwhile, the methylation levels of HOXAl decreased after treatment with DNA methyltransferase inhibitor 5-Aza-2-deoxycytidine (5-Aza-CdR) (Figure 3a). Subsequently, the $\mathrm{IC}_{50}$ values of H69AR and H446AR cells decreased in response to CDDP, ADM, and VP-16 after treatment by 5-Aza-CdR (Figure 3b). Meanwhile, chemosensitivity was measured by CCK-8 assays when resistant cell lines (H69AR and H446AR) were treated with siHOXA1 and 5-Aza-CdR. The results showed that there is no significant difference between a

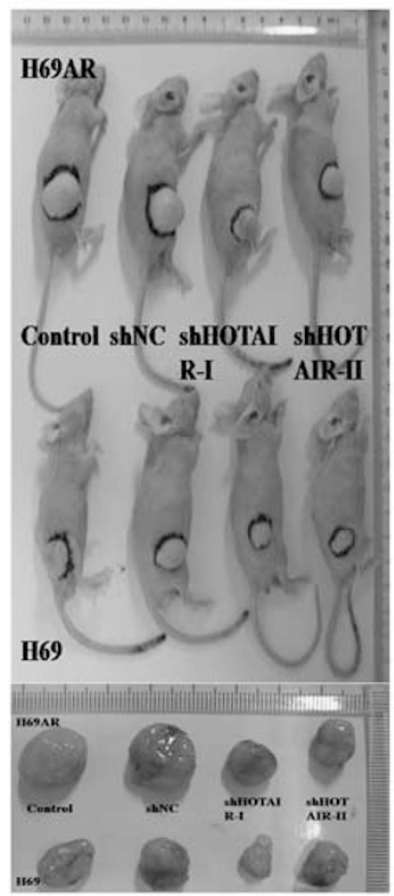

b
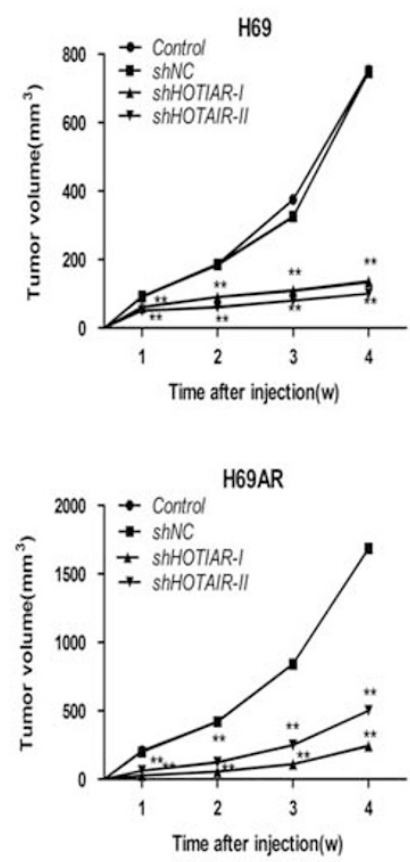

C

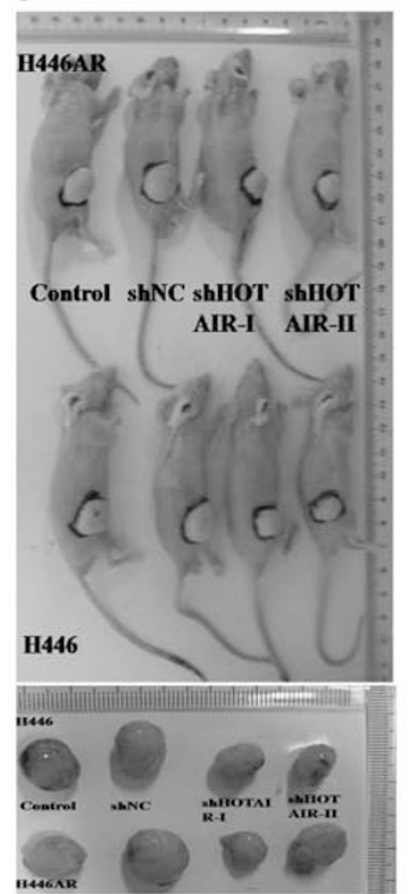

d
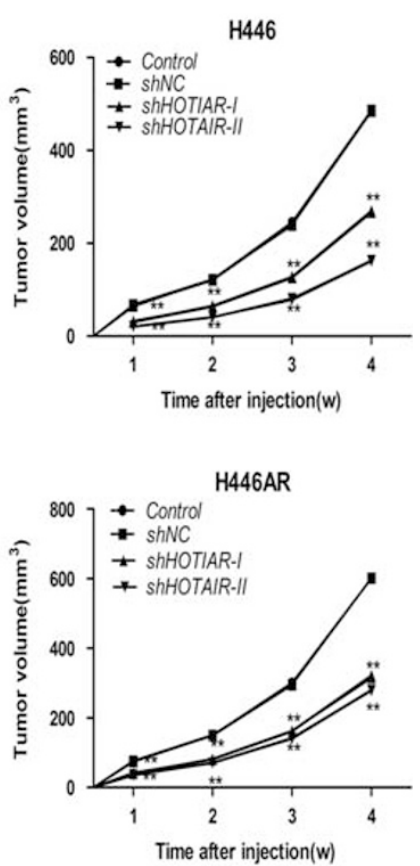

Figure 2 LncRNA-HOTAIR suppressed tumor growth in nude mice subcutaneously inoculated with shHOTAIR-treated cells compared with shNC-treated cells. (a) H69 and H69AR cells; (c) H446 and H446AR cells. (b and d) The curves of tumor growth. The tumor volume was calculated using the ellipsoid volume formula $\left(0.5 \times I \times w^{2}\right.$, where $I$ is length and $w$ is width) every week. ${ }^{*} P<0.01$. 

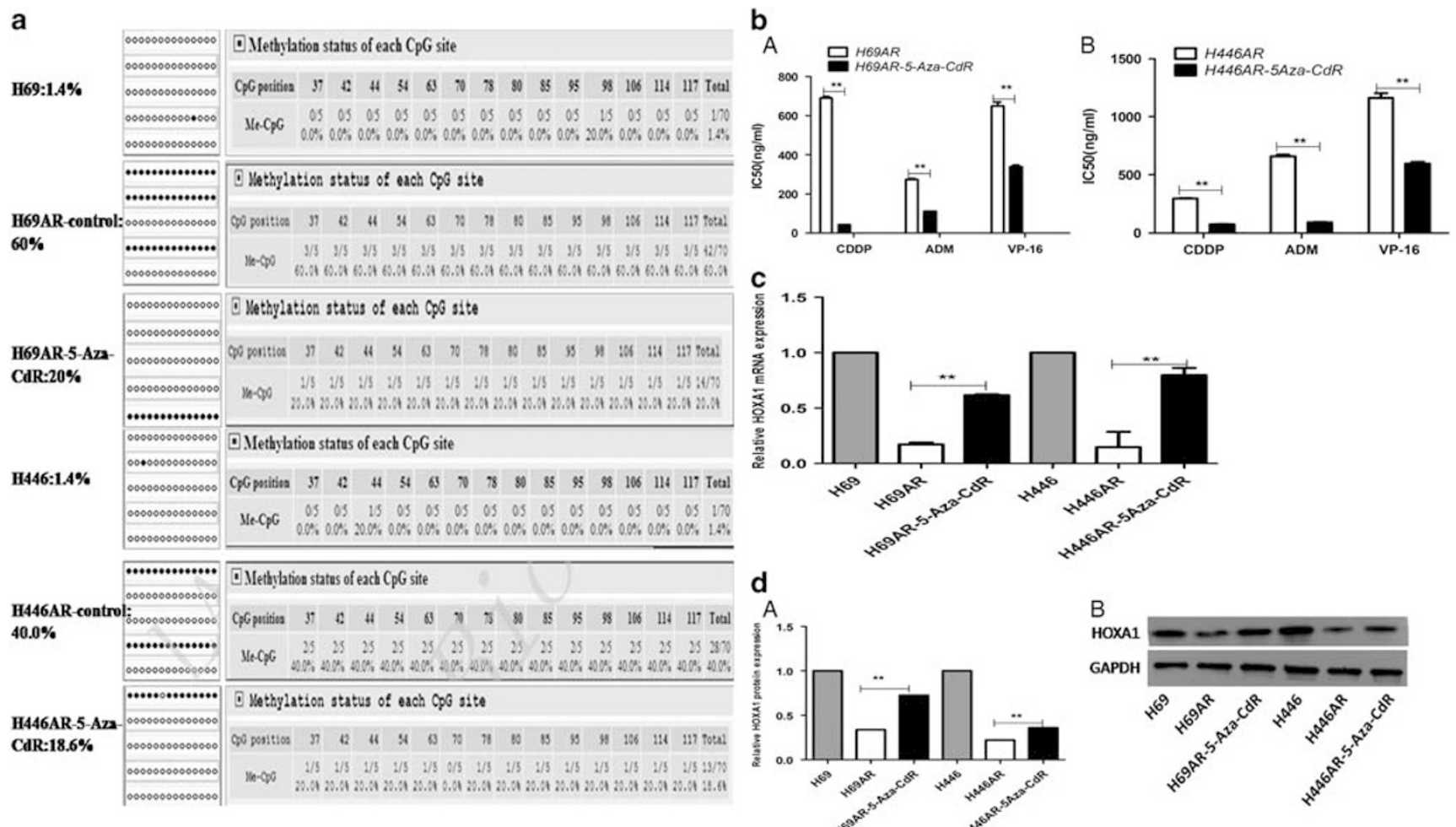

Figure 3 Methylation status of HOXA1 was associated with multidrug resistance. (a) Analysis of the HOXA1 CpG islands in H69/H69AR/5-Aza-CdRtreated H69AR cells (top set) and H446/H446AR/5-Aza-CdR-treated H446AR cells (bottom set) by bisulfite sequencing PCR. (b) IC 50 of H69AR and H446AR cells to anticancer drugs after demethylation with 5 -Aza-CdR. Results are mean \pm s.d. ${ }^{* *} P<0.01$. (c and d) HOXA1 expression was measured by RT-PCR and western blot after treatment with 5-Aza-CdR.

control groups and siHOXA1-5-Aza-CdR groups in H446AR cells upon CDDP and ADM treatment (Supplementary Figures S5 and S6). The mRNA and protein expressions of HOXA1 in H69AR and H446AR with treatment of 5-Aza-CdR were upregulated by 4 -15-fold and 2-3-fold, respectively (Figure 3c and d). The results suggested that aberrant methylation of HOXA1 may be involved in multidrug resistance of SCLC.

\section{HOTAIR Associated with HOXA1}

We performed bioinformatics-based tool (catRAPID algorithm) to predict the interaction between lncRNA-HOTAIR and HOXA1, and found the highest interaction sore (normalized) was 3.68 (location: lncRNA-HOTAIR region: 349-444, HOXA1 region: 226-277) (Figure 4a and Supplementary Table S1). RIP method was further carried out to show a significant enrichment of IncRNA-HOTAIR with the HOXA1 antibody as compared with the nonspecific IgG control antibody (Figure 4b and Supplementary Figure S4). Next, we observed the upregulation of the HOXA1 mRNA expression by $12.7-886.3$-fold and protein expression by $12.5-24.9$-fold in the lncHOTAIR-downregulated cells (Figure $4 \mathrm{c}$ and d).

\section{LncRNA-HOTAIR Epigenetically Silenced HOXA1 through DNMT1 and DNMT3b}

We found the methylation level of HOXA1 decreased consistently with lncRNA-HOTAIR downregulation (Figure 5a).
To better characterize the way by which IncRNA-HOTAIR regulated the HOXA1 methylation, we performed ELISA assay to measure the expression of DNMTs (DNMT1, DNMT3a, and DNMT 3b). The results revealed that both DNMT1 and DNMT3b expression decreased significantly in H69AR and H446AR cells after transfection with siHOTAIR, but no significant difference was found in the DNMT3a expression (Figure 5b). Therefore, these findings suggested that loss of lncRNA-HOTAIR demethylated HOXA1 by decreasing expression of DNMT1 and DNMT3b.

\section{DISCUSSION}

Recently, forced expression of lncRNA-HOTAIR has emerged as a powerful predictor of eventual metastasis and chemoresistance in several kinds of tumors such as colorectal cancer, sarcoma, and lung adenocarcinoma. ${ }^{7,19-22}$ However, the role of IncRNA-HOTAIR in the chemoresistance of SCLC remains unknown. In this study, we initially identified IncRNAHOTAIR as a regulator in chemoresistance of SCLC by qRT-PCR in H69/H69AR and H446/H446AR cells. We further demonstrated that downregulation of IncRNAHOTAIR led to increased cell sensitivity to CDDP, ADM, and VP-16, enhanced cell apoptosis and cell cycle arrest, and reduced tumor growth in vivo. To our knowledge, this is the first report to investigate the function of lncRNA-HOTAIR in mediating chemoresistance of SCLC. 
Some previous studies have shown a relationship between gene silencing by targeted DNA methylation and chemotherapy response to SCLC. For example, DNA methylation of caspase-8 in SCLC cells resulted in resistance to tumor necrosis factor-related apoptosis-inducing ligand (TRAIL). ${ }^{23}$ We found that downregulation of HOXA1 gene affects SCLC cell survival and chemoresistance before. ${ }^{16}$ In our study, BSP method showed hypermethylation status of HOXA1 CpG islands in the resistant cells as compared with the parental cells. The methylation level of HOXA1 became lower and the expression level was improved remarkably when H69AR and $\mathrm{H} 446 \mathrm{AR}$ cells were treated with $5-\mathrm{Aza}-\mathrm{CdR}$. Then, the $\mathrm{IC}_{50}$ values of H69AR and H446AR cells became lower in response to CDDP, ADM, and VP-16 after treatment with 5-Aza-CdR. To further investigate whether aberrant methylation of HOXA1 is necessary in multidrug resistance, we knocked down HOXA1 in the context of 5-aza-CdR treatment, and the results showed that there is no significant difference between control groups and siHOXA1-5-Aza-CdR groups in H446AR cells upon CDDP and $\mathrm{ADM}$ treatment. In addition to caspase- 8 reported by Kaminskyy et al, ${ }^{23}$ our results suggested DNA methylation of HOXA1 was closely associated with chemoresistance of SCLC.

LncRNA has been described to mediate maintenance of DNA methylation and transcriptionally silence proteincoding genes. ${ }^{24}$ Therefore, we sought to investigate whether lncRNA-HOTAIR was involved in regulating HOXA1 methylation. Using bioinformatics-based tools (catRAPID algo- rithm), we found there was a possible interaction between lncRNA-HOTAIR and HOXA1. Next, we observed that the methylation level of HOXA1 was obviously consistent with lncRNA-HOTAIR downregulation. RIP method was subsequently carried out to validate the interaction between IncRNA-HOTAIR and HOXA1. These results support that lncRNA-HOTAIR can affect the methylation level of HOXA1.

DNA methylation is catalyzed via DNMTs: DNMT1, DNMT3a, and DNMT3b. DNMT1 is the most abundant DNA methyltransferase in mammalian cells and codes for maintenance methylation and de novo methylation. DNMT3a and DNMT3b are responsible for catalyzing de novo methylation during early embryogenesis, thereby establishing a somatic methylation pattern. ${ }^{25}$ To better understand the way by which IncRNA-HOTAIR regulated the HOXA1 methylation level, we measured DNMT expression including DNMT1, DNMT3a, and DNMT3b in H69AR and H446AR cells by ELISA assay. The results showed that the expression of DNMT1 and DNMT3b decreased significantly after the downregulation of IncRNA-HOTAIR, but no significant difference was observed in DNMT3a expression. Previous studies have shown that genetic disruption of both DNMT1 and DNMT3b nearly eliminated methyltransferase activity, and reduced genomic DNA methylation by $>95 \%$ in colorectal cancer cells. ${ }^{26}$ Inhibiting DNMTs by 5 -Aza-CdR treatment led to significant inhibition of expression of DNMT1 and DNMT3b but not DNMT3a and enhanced expression of PTEN and p21 analyzed in glioma. ${ }^{27}$ All these
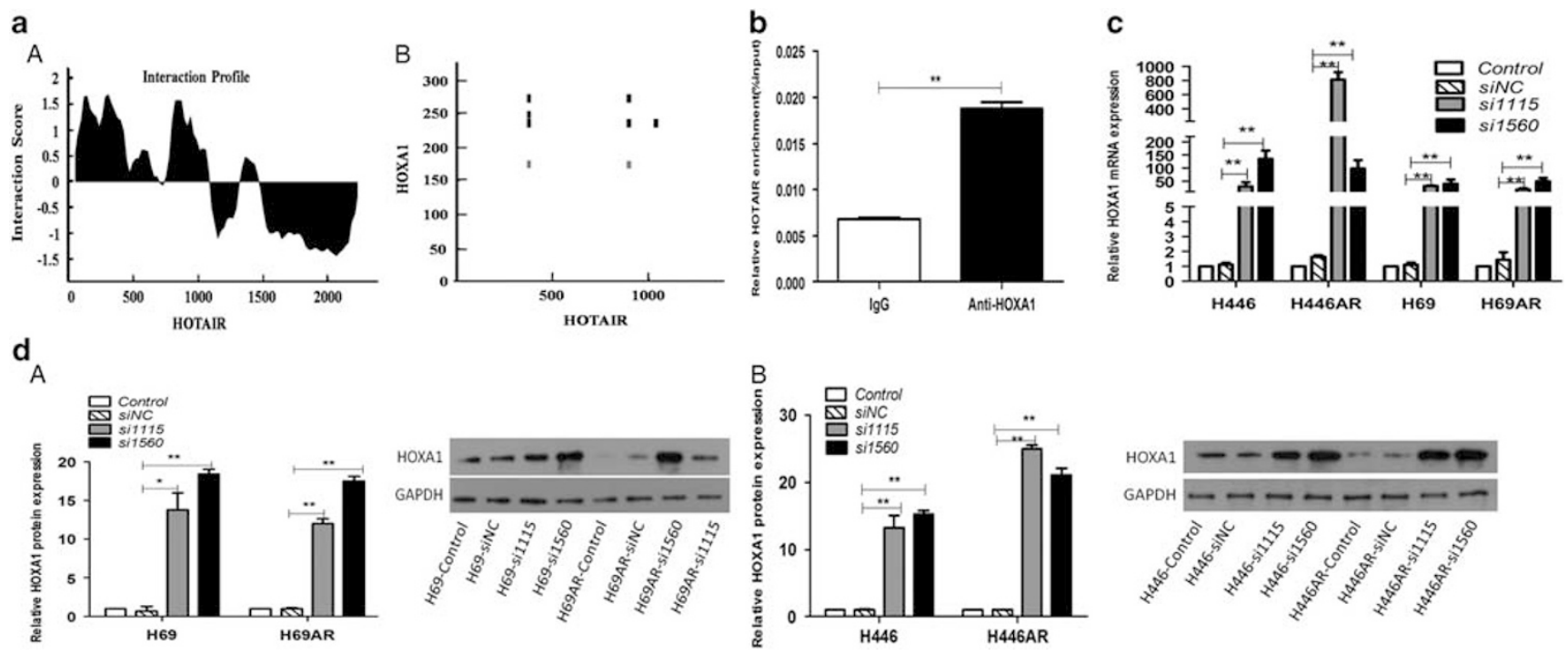

Figure 4 HOXA1 expression was regulated by InCRNA-HOTAIR. (a) Bioinformatics prediction of the interaction between IncRNA-HOTAIR and HOXA1. (b) RIP enrichment was determined as RNA associated with HOXA1 antibody relative to lgG negative control. (c and d) The mRNA and protein expression of HOXA1 after cells were transfected with siHOTAIR. ${ }^{*} P<0.05,{ }^{* *} P<0.01$.

Figure 5 LncRNA-HOTAIR epigenetically silenced HOXA1 through DNMT1 and DNMT3b. (a) HOXA1 methylation status of H69/H69AR/siHOTAIR-treated H69AR cells (top set) and H446/H446AR/siHOTAIR-treated H446AR cells (bottom set). (b) Quantification of DNMTs after transfection with siHOTAIR in both resistant cells by ELISA. Results are mean \pm s.d. ${ }^{*} P<0.01$. 
a

H69:1.4\%

00000000000000

00000000000000

00000000000000

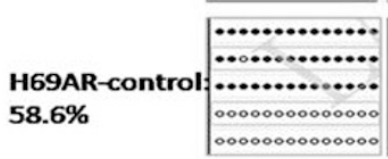

H69AR-siNC:
57.1\%

00000000000000

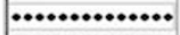

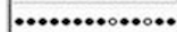

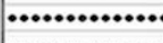

00000000000000

00000000000000

H69AR-si1115:

$2.9 \%$

00000000000000

00000000000000

00000000000000

00000000000000

H69AR-si1560:
\%\%

000000000000000

00000000000000

00000000000000

000000000000000

00000000000000

H446:1.4\%

00000000000000

00000000000000

00000000000000

00000000000000

H446AR-contro

$40.0 \%$

00000000000000

H446AR-siNC: $38.57 \%$
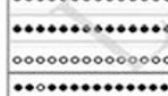

.000.0.1.1.

0000000000000000

000000000000000

(................

00000000000000

000000000000000

H446AR-si1115:

2.86\%

00000000000000

00000000000000

|

H446AR-si1560:

\%\%

0

00000000000000

00000000000000
Methylation status of each $\mathrm{CpG}$ site

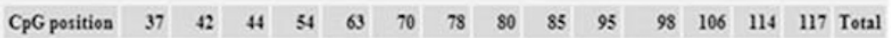

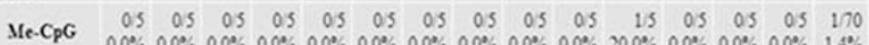
Me-CpG $\quad 0.0 \%, 0.0 \% \quad 0.0 \% \quad 0.0 \% \quad 0.0 \% \quad 0.0 \% \quad 0.0 \% \quad 0.0 \% \quad 0.0 \% \quad 0.0 \% \quad 20.0 \% \quad 0.0 \% \quad 0.0 \% \quad 0.0 \% \quad 1.4 \%$

- Methylation status of each CpG site

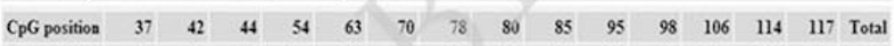

$\begin{array}{llllllllllllllll}0.5 & 35 & 25 & 35 & 35 & 35 & 3.5 & 35 & 3.5 & 35 & 3.5 & 3.5 & 3.5 & 35 & 4170\end{array}$

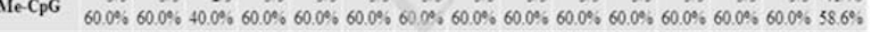

- Methylation status of each $\mathrm{CpG}$ site

$\begin{array}{llllllllllllllll}\text { CpG position } & 37 & 42 & 44 & 54 & 63 & 70 & 78 & 80 & 85 & 95 & 98 & 106 & 114 & 117 & \text { Total }\end{array}$

$\begin{array}{llllllllllllllll}\text { Ve-CpG } & 3 / 5 & 3 / 5 & 3 / 5 & 3 / 5 & 3 / 5 & 3 / 5 & 3 / 5 & 3 / 5 & 25 & 3 / 5 & 3 / 5 & 25 & 3 / 5 & 3 / 5 & 4070\end{array}$

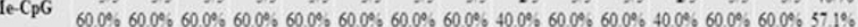

- Methylation status of each CpG site

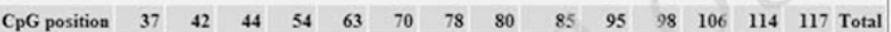

$\begin{array}{llllllllllllllll}\text { Me-CpG } & 0.5 & 0.5 & 0.5 & 0.5 & 0.5 & 0.5 & 0,5 & 0.5 & 2.5 & 0.5 & 0.5 & 0.5 & 0.5 & 0.5 & 270\end{array}$

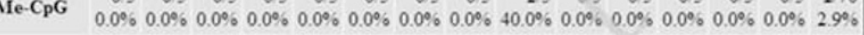

- Methylation status of each CpG site

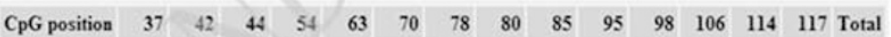

$\begin{array}{llllllllllllllll}\text { Me-CpG } & 0.5 & 0.5 & 0.5 & 0.5 & 0.5 & 0.5 & 0.5 & 0.5 & 0.5 & 0.5 & 0.5 & 0.5 & 0.5 & 0.5 & 0.70\end{array}$ $0.0 \% 0.0 \% \quad 0.0 \% \quad 0.0 \% \quad 0.0 \% \quad 0.0 \% \quad 0.0 \% \quad 0.0 \% \quad 0.0 \% \quad 0.0 \% \quad 0.0 \% \quad 0.0 \% \quad 0.0 \% \quad 0.0 \% \quad 0.0 \%$

- Methylation status of each $C_{p} G$ site

\begin{tabular}{|l|l|l|l|l|l|l|l|l|l|l|l|l|l|l|l|}
\hline CpG position & 37 & 42 & 44 & 54 & 63 & 70 & 78 & 80 & 85 & 95 & 98 & 106 & 114 & 117 & Total \\
\hline
\end{tabular}

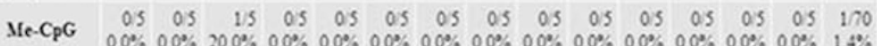
$0.0 \% \quad 0.0 \% \quad 20.0 \% \quad 0.0 \% \quad 0.0 \% \quad 0.0 \% \quad 0.0 \% \quad 0.0 \% \quad 0.0 \% \quad 0.0 \% \quad 0.0 \% \quad 0.0 \% \quad 0.0 \% \quad 0.0 \% \quad 1.4 \%$

- Methylation status of each $\mathrm{CpG}$ site

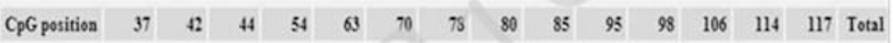
$\begin{array}{llllllllllllllll}\text { Me-CpG } & 25 & 25 & 25 & 25 & 25 & 25 & 25 & 25 & 25 & 25 & 25 & 25 & 25 & 25 & 2870\end{array}$

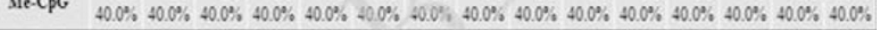
Methylation status of each $\mathrm{CpG}$ site

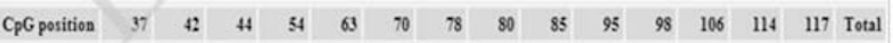
$\begin{array}{llllllllllllllll}\text { Me-CpG } & 25 & 25 & 15 & 25 & 25 & 25 & 25 & 25 & 25 & 25 & 25 & 25 & 25 & 25 & 27770\end{array}$

Methylation status of each $\mathrm{CpG}$ site

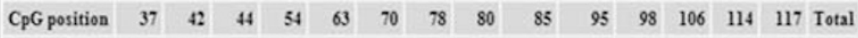

$\begin{array}{llllllllllllllll}\text { Me-CpG } & 0.5 & 0.5 & 0.5 & 0.5 & 0.5 & 0.5 & 0.5 & 0.5 & 1 / 5 & 1 / 5 & 0.5 & 0.5 & 0.5 & 0.5 & 270\end{array}$

$0.0 \% \quad 0.0 \% \quad 0.0 \% \quad 0.0 \% \quad 0.0 \% \quad 0.0 \% \quad 0.0 \% \quad 0.0 \% \quad 20.0 \% \quad 20.0 \% \quad 0.0 \% \quad 0.0 \% \quad 0.0 \% \quad 0.0 \% \quad 2.9 \%$

\section{Methylation status of each $\mathrm{CpG}_{\mathrm{p}}$ site}

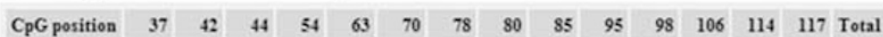

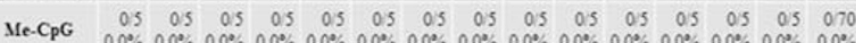
Me-CpG $\quad 0.0 \% \quad 0.0 \%, 0.0 \%, 0.0 \%, 0.0 \% \quad 0.0 \% \quad 0.0 \%, 0.0 \% \quad 0.0 \%, 0.0 \% \quad 0.0 \% \quad 0.0 \% \quad 0.0 \% \quad 0.0 \% \quad 0.0 \%$ b

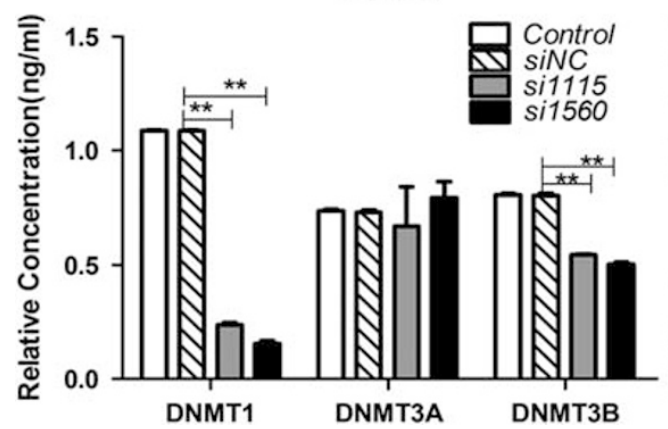

H69AR

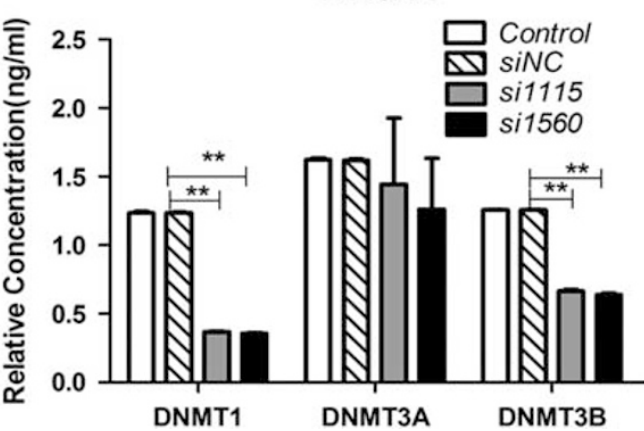



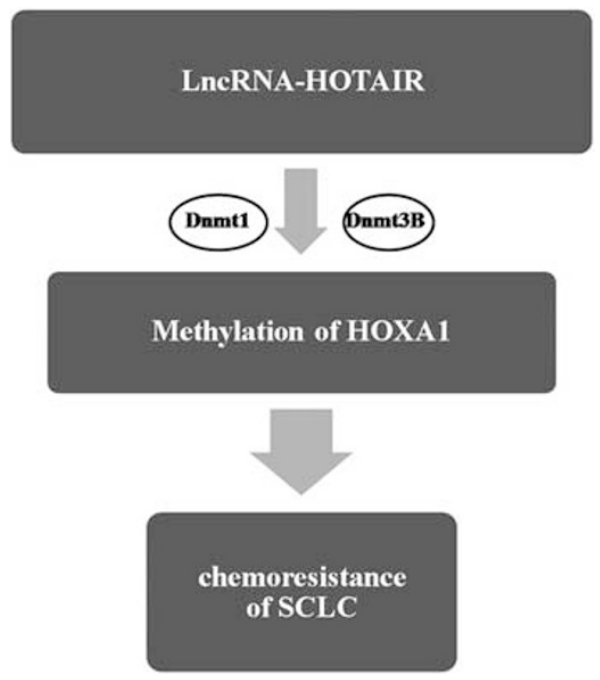

Figure 6 Flow diagram of the IncRNA-HOTAIR on SCLC chemoresistance by regulating HOXA1 methylation.

results including ours suggested that DNMT1 and DNMT3b cooperatively maintained DNA methylation and gene silencing in oncogenesis and chemoresistance of tumors.

In summary, our results demonstrated that lncRNAHOTAIR was overexpressed in the resistant cells. Downregulation of lncRNA-HOTAIR led to increased cell sensitivity to anticancer drugs, enhanced cell apoptosis and cell cycle arrest, and reduced tumor growth in vivo. DNA methylation of HOXA1 was required for the chemoresistance of SCLC. LncRNA-HOTAIR epigenetically silenced HOXA1 through DNMT1 and DNMT3b. Our findings revealed that IncRNA-HOTAIR can modulate chemoresistance of SCLC by regulating HOXA1 methylation (Figure 6). LncRNAHOTAIR might be a predictor for chemotherapy response and a therapeutic target against chemoresistance of SCLC.

Supplementary Information accompanies the paper on the Laboratory Investigation website (http://www.laboratoryinvestigation.org)

\section{ACKNOWLEDGMENTS}

This work was supported by the National Natural Science Foundation of China (81372508) and the National University Student Innovation Program (201312121009)

\section{DISCLOSURE/CONFLICT OF INTEREST}

The authors declare no conflict of interest.

1. Van Meerbeeck JP, Fennell DA, De Ruysscher DK. Small-cell lung cancer. Lancet 2011;378:1741-1755.

2. Puglisi $M$, Dolly $S$, Faria $A$ et al. Treatment options for small cell lung cancer - do we have more choice? Br J Cancer 2010;102:629-638.

3. Guttman M, Rinn JL. Modular regulatory principles of large noncoding RNAs. Nature 2012;482:339-346.

4. Spizzo R, Almeida MI, Colombatti $A$ et al. Long non-coding RNAs and cancer: a new frontier of translational research? Oncogene 2012;31: 4577-4587.

5. Yang $\mathrm{Y}, \mathrm{Li} \mathrm{H}$, Hou $\mathrm{S}$ et al. The noncoding RNA expression profile and the effect of IncRNA AK126698 on cisplatin resistance in non-small-cell lung cancer cell. PloS One 2013;8:e65309.
6. Kogo $\mathrm{R}$, Shimamura $\mathrm{T}$, Mimori $\mathrm{K}$ et al. Long noncoding RNA HOTAIR regulates polycomb-dependent chromatin modification and is associated with poor prognosis in colorectal cancers. Cancer Res 2011;71:6320-6326.

7. Gupta RA, Shah N, Wang KC et al. Long non-coding RNA HOTAIR reprograms chromatin state to promote cancer metastasis. Nature 2010;464:1071-1076.

8. Ono $\mathrm{H}$, Motoi $\mathrm{N}$, Nagano $\mathrm{H}$ et al. Long noncoding RNA HOTAIR is relevant to cellular proliferation, invasiveness, and clinical relapse in small-cell lung cancer. Cancer Med 2014;3:632-642.

9. Heyn $\mathrm{H}$, Esteller M. DNA methylation profiling in the clinic: applications and challenges. Nat Rev Genet 2012;13:679-692.

10. Tessema $M$, Yingling $C M$, Thomas $C L$ et al. SULF2 methylation is prognostic for lung cancer survival and increases sensitivity to topoisomerase-I inhibitors via induction of ISG15. Oncogene 2012;31: 4107-4116.

11. Liu XH, Sun $M$, Nie FQ et al. Lnc RNA HOTAIR functions as a competing endogenous RNA to regulate HER2 expression by sponging miR-331$3 p$ in gastric cancer. Molecular Cancer 2014;13:92.

12. Li X, Wu Z, Mei $Q$ et al. Long non-coding RNA HOTAIR, a driver of malignancy, predicts negative prognosis and exhibits oncogenic activity in oesophageal squamous cell carcinoma. $\mathrm{Br} J$ Cancer 2013;109:266-2278.

13. Ibanez de Caceres I, Cortes-Sempere M, Moratilla C et al. IGFBP-3 hypermethylation-derived deficiency mediates cisplatin resistance in non-small-cell lung cancer. Oncogene 2010;29:1681-1690.

14. Cortes-Sempere M, de Miguel MP, Pernia O et al. IGFBP-3 methylationderived deficiency mediates the resistance to cisplatin through the activation of the IGFIR/Akt pathway in non-small cell lung cancer. Oncogene 2013;32:1274-1283.

15. Plowright $\mathrm{L}$, Harrington $\mathrm{KJ}$, Pandha HS et al. HOX transcription factors are potential therapeutic targets in non-small-cell lung cancer (targeting HOX genes in lung cancer). Br J Cancer 2009;100:470-475.

16. Xiao F, Bai $Y$, Chen $Z$ et al. Downregulation of HOXA1 gene affects small cell lung cancer cell survival and chemoresistance under the regulation of miR-100. Eur J Cancer 2014;50:1541-1554.

17. Mohankumar KM, Perry JK, Kannan N et al. Transcriptional activation of signal transducer and activator of transcription (STAT) 3 and STAT5B partially mediate homeobox A1-stimulated oncogenic transformation of the immortalized human mammary epithelial cell. Endocrinology 2008;149:2219-2229.

18. Mohankumar KM, Xu XQ, Zhu T et al. HOXA1-stimulated oncogenicity is mediated by selective upregulation of components of the p44/42 MAP kinase pathway in human mammary carcinoma cells. Oncogene 2007;26:3998-4008.

19. Zha TZ, Hu BS, Yu HF et al. Overexpression of HOXA1 correlates with poor prognosis in patients with hepatocellular carcinoma. Tumour Biol 2012;33:2125-2134.

20. Zhou $Y$, Sun $Y$, Guo L. Establishment of an adriamycin multi-drug resistant human small cell lung cancer cell line and its correlationship with the expression of Bcl-2 family proteins. Shandong Med 2009;49: $31-34$.

21. Milhem MM, Knutson T, Yang $S$ et al. Correlation of MTDHAEG-1 and HOTAIR expression with metastasis and response to treatment in sarcoma patients. J Cancer Sci Ther 2011;S5: 004.

22. Liu Z, Sun M, Lu K et al. The long noncoding RNA HOTAIR contributes to cisplatin resistance of human lung adenocarcinoma cells via downregulation of p21(WAF1/CIP1) expression. PloS One 2013;8:e77293.

23. Kaminskyy VO, Surova OV, Vaculova A et al. Combined inhibition of DNA methyltransferase and histone deacetylase restores caspase-8 expression and sensitizes SCLC cells to TRAlL. Carcinogenesis 2011;32: $1450-1458$

24. Mohammad F, Pandey GK, Mondal T et al. Long noncoding RNAmediated maintenance of DNA methylation and transcriptional gene silencing. Development 2012;139:2792-2803.

25. Okano M, Bell DW, Haber DA et al. DNA methyltransferases Dnmt3a and Dnmt3b are essential for de novo methylation and mammalian development. Cell 1999;99:247-257.

26. Rhee I, Bachman KE, Park BH et al. DNMT1 and DNMT3b cooperate to silence genes in human cancer cells. Nature 2002;416:552-556.

27. Rajendran G, Shanmuganandam K, Bendre $A$ et al. Epigenetic regulation of DNA methyltransferases: DNMT1 and DNMT3B in gliomas. J Neuroendocrinol 2011;104:483-494. 